\section{Schein-Praxisgemeinschaft führt zu Honorarkürzung}

_ Nach einem Urteil des Landessozialgerichts (LSG) Niedersachsen-Bremen sind sachlich-rechnerische Honorarberichtigungen durch die $\mathrm{KV}$ möglich, wenn eine als Berufsausübungsgemeinschaft (BAG) betriebene, fachgleiche Vertragsarztkooperation nach außen hin in eine getrennt abrechnende Praxisgemeinschaft umgewandelt wird, dabei jedoch die für eine BAG kennzeichnende gemeinsame Behandlung des Patientenstamms unverändert fortgeführt wird (Az.: L 3 KA 16/14). In einem solchen Fall kann laut dem Gericht eine missbräuchliche Steigerung der Fallzahl und der Abrechnung vorliegen. Die Grenze dafür sei erreicht, wenn im Quartal 20\% der Patienten in den einzel- nen Praxen identisch sind. Das entspricht dem Orientierungswert für Abrechnungsauffälligkeiten in der Plausibilitätsprüfung.

\section{MMW-KOMMENTAR}

Im konkreten Fall bestand die Praxisgemeinschaft aus insgesamt drei Ärzten, die in einer Einzelpraxis und einer BAG tätig waren. Die kleinere Einzelpraxis hatte durchgehend deutlich mehr als 20\% Patienten, die auch in der kooperierenden BAG behandelt wurden, und überschritt damit den Grenzwert. Die $B A G$, die insgesamt mehr Patienten hatte, lag teilweise unter der Grenze.

Allerdings erachtete das LSG es für sachwidrig und unvereinbar mit dem Gleichbehandlungsgebot, allein die kleinere Praxis in Haf-

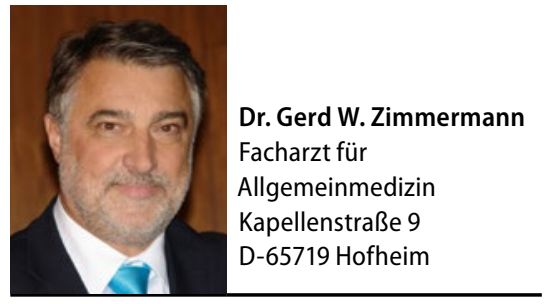

tung zu nehmen. Es bestimmte deshalb, dass die Orientierungswerte für den Anteil identischer Fälle modifiziert werden müssen, wenn es um das Behandlungsverhalten einer Praxisgemeinschaft geht, die aus einer Einzelpraxis und einer BAG besteht.

Die Richter sahen es auch nicht als entlastend an, dass die BAG teils unter die 20\%-Marke gesunken war. Sie wiesen darauf hin, das der für BAG übliche Wert von maximal 15\% stets übertroffen wurde.

Eine Honorarberichtigung kann somit auch ohne Vorliegen eines bestimmten Mindestanteils von gemeinsamen Patienten erfolgen, wenn ohne Zweifel feststeht, dass Vertragsärzte in der Struktur einer BAG gearbeitet und nur nach außen das Bild einer Praxisgemeinschaft erweckt haben.

\title{
GOÄ: Bei der Sono kein Geld verschenken
}

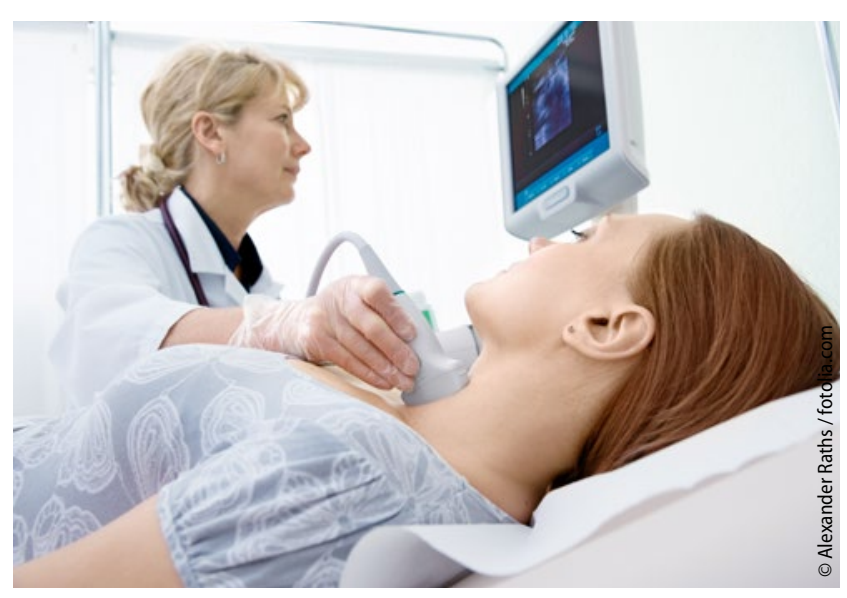

Für die Schilddrüsen-Sonografie gibt es eine eigene Ziffer.

_ Die Ultraschalluntersuchung eines Organs wird in der GOÄ nach Nr. 410 abgerechnet und ist mit 200 Punkten bewertet. Als Organ gilt dabei ein zusammenhängender Körperteil, der eine Einheit mit bestimmten Funktionen bildet. Paarige Organe wie die Nieren stellen deshalb jeweils ein eigenes Organ dar. Auch Gallenblase, Gallenwege, Hoden, Nebenhoden, Speicheldrüsen, Schilddrüse, Epithelkörperchen der Nebenschilddrüse sind jeweils eigene Organe. Für die Untersuchung eines weiteren Organs kann zusätzlich die Nr. 420 berechnet werden, allerdings nur bis zu dreimal pro Sitzung,

\section{MMW-KOMMENTAR}

Für die Sonografie der Schilddrüse gibt es eine eigene Ziffer: die Nr. 417, die mit 210 Punkten etwas höher bewertet ist. Werden in einer Sitzung also sowohl die Schilddrüse als auch andere Organe geschallt, setzt man die Nr. 417 als Leitnummer an und rechnet die anderen Organe nach Nr. 420 ab. Honorartechnisch ist eine solche Selektion nicht zu beanstanden.

Beachtenswert ist noch, dass die echokardiografischen Untersuchungen nach den Nrn. 422-424 in gleicher Sitzung neben den Nrn. 410, 417 und 420 berechnet werden können. Lediglich wenn der Zuschlag für die Anwendung des Duplex-Verfahrens nach Nr. 401 abgerechnet wird, ist der parallele Ansatz der Nrn. 422-424 ausgeschlossen. 